\title{
APPLICATIONS OF GENERAL MONOTONE SEQUENCES AND FUNCTIONS TO TRIGONOMETRIC SERIES AND INTEGRALS
}

\author{
PÉTER KóRUs
}

Abstract. Using the concept of general monotone sequences and functions we were able to extend some of the results of L. Leindler from sine series to cosine series and to sine and cosine integrals as well.

Mathematics subject classification (2010): 40A05, 40A10, 42A16, 42A20, 42A32, 42A38.

Keywords and phrases: general monotone sequences, general monotone functions, trigonometric series, trigonometric integrals.

\section{REFERENCES}

[1] M. Dyachenko, E. Liflyand And S. Tikhonov, Uniform convergence and integrability of Fourier integrals, J. Math. Anal. Appl. 372, 1 (2010), 328-338.

[2] M. DYACHENKO AND S. TIKHONOV, Integrability and continuity of functions represented by trigonometric series: coefficients criteria, Studia Math. 193, 3 (2009), 285-306.

[3] L. LeIndLER, A new extension of monotone sequences and its applications, J. Inequal. Pure and Appl. Math. 7, 1 (2006), Art. 39.

[4] L. LeINDLER, Newer applications of generalized monotone sequences, J. Inequal. Pure and Appl. Math. 7, 5 (2006), Art. 167.

[5] E. LiflyAnd And S. TiKhonov, The Fourier transforms of general monotone functions, in: Analysis and Mathematical Physics, in: Trends Math., Birkhäuser, 2009, pp. 377-395.

[6] F. Móricz, On the uniform convergence of sine integrals, J. Math. Anal. Appl. 354 (2009), $213-219$.

[7] S. A. Telyakovskiľ, On partial sums of Fourier series of functions of bounded variation, Proc. Steklov Inst. Math. 219 (1997), 372-381. 